\title{
Anmeldung zur DINI-Jahrestagung 2019 eröffnet
}

\author{
Die Jahrestagung der Deutschen Initiative für Netzwerk- \\ information (DINI) widmet sich vom 8. bis 9. Oktober 2019 \\ in Osnabrück aktuellen Handlungsfeldern der Digitalisierung \\ von Forschung und Lehre
}

http://doi.org/10.1515/bd-2019-0077

Im Mittelpunkt der diesjährigen Jahrestagung der Deutschen Initiative für Netzwerkinformation (DINI) steht das Thema „Künstliche Intelligenz in Forschung und Lehre“.

Vom 8. bis 9. Oktober 2019 werden sich dazu Expertinnen und Experten aus der Wissenschaft und ihren Informationsinfrastrukturen an der Universität Osnabrück austauschen. Die Anmeldung zur Tagung ist ab sofort möglich.

In diesem Jahr feiert die Deutsche Initiative für Netzwerkinformation (DINI) ihr 20-jähriges Bestehen. Seit 1999 vernetzt die Initiative wissenschaftliche Bibliotheken, Medienzentren und Rechenzentren sowie weitere Wissenschaftseinrichtungen und -organisationen in Deutschland und fördert die Entwicklung digitaler Dienstleistungen und Informationsinfrastrukturen für die digital arbeitende Wissenschaft. Das Jubiläum nimmt DINI zum Anlass, unter dem Motto „1999-2019-2039: Innovative Projekte in Forschung, Lehre und Infrastruktur" über Stand und Zukunft der Digitalisierung von Forschung und Lehre zu diskutieren.

Eine Postersession wird Projekten und Institutionen neben den eingeladenen Vorträgen Gelegenheit geben, sich vorzustellen und mit Teilnehmenden ins Gespräch zu kommen. Das Programmkomitee bittet um die Einreichung von Abstracts für Poster, die Projekte und Initiativen mit Bezug zum Motto „Innovative Projekte in Forschung, Lehre und Infrastruktur“" vorstellen.

Im Rahmen der Jahrestagung findet auch die Preisverleihung des studentischen Wettbewerbs „Lernen 4.0 - Gestalte Deinen Lernraum“ statt. Der Wettbewerb für neuartige Lernkonzepte an Hochschulen und Universitäten sammelt zum fünften Mal Ideen zur Verbesserung des digital unterstützten Lehrens und Lernens.

Lokaler Veranstalter der DINI-Jahrestagung ist die Universitätsbibliothek Osnabrück. 


\section{Weitere Informationen im Internet:}

- Zur DINI-Jahrestagung: https://dini.de/veranstaltungen/jahrestagungen/20dini-jahrestagung-2019

- Zur Poster-Session: https://dini.de/veranstaltungen/jahrestagungen/20-dinijahrestagung-2019/call-for-posters

- Zum DINI-Wettbewerb: https://dini.de/wettbewerbe/lernen-40-gestalte-dei nen-lernraum

- DINI-Homepage: https://dini.de

\section{Über die Deutsche Initiative für Netzwerkinformation (DINI):}

Die Deutsche Initiative für Netzwerkinformation (DINI) e. V. ist der überregionale Zusammenschluss von wissenschaftlichen Bibliotheken, Medienzentren, Rechenzentren und Fachgesellschaften in Forschung und Lehre in Deutschland. DINI ist Partner von Hochschulen und außeruniversitären Forschungseinrichtungen bei der Weiterentwicklung der Informationsinfrastrukturen. 